\title{
LXI. The forms of planetary orbits on the theory of relativity
}

\section{W.B. Morton M.A.}

To cite this article: W.B. Morton M.A. (1921) LXI. The forms of planetary orbits on the theory of relativity, Philosophical Magazine Series 6, 42:250, 511-522, DOI: 10.1080/14786442108633793

To link to this article: http://dx.doi.org/10.1080/14786442108633793

曲 Published online: 08 Apr 2009.

Submit your article to this journal $\pi$

Џ Article views: 3

Q View related articles $\longleftarrow$

Citing articles: 2 View citing articles 5 
Forms of Planetary Orbits on Theory of Relativity. 511 We have also

$$
\frac{\lambda}{\mu^{2}} \cdot \frac{d \mu}{d \lambda}=-\frac{\lambda^{2}}{\mu^{3}}\left(c+\underset{\left(\lambda^{2}-\beta^{2}\right)^{2}}{b}\right) .
$$

Using these formulæ, I have constructed the following table similar to that on p. 44 of "Relativity and the Electron 'Theory.'

\begin{tabular}{|c|c|c|c|c|}
\hline Wave-length $\times 10^{3}$ & $\kappa_{\mathrm{F}}$. & $\kappa_{\mathrm{A}}$. & $\kappa_{\mathrm{C}}$. & $\kappa_{\mathrm{Z}}$. \\
\hline 4500 & 4424 & 4615 & 4615 & +465 \\
\hline 4580 & $\cdot 44 \cdot 0$ & 4579 & $\cdot 4634$ & $\cdot 46: 3$ \\
\hline 5461 & $-488: 3$ & $\cdot 4501$ & $\cdot 4541$ & 451 \\
\hline 6870 & -4315 & 4438 & 4464 & $44 \vec{i}$ \\
\hline
\end{tabular}

In this table $\kappa_{F}$ is the convection coefficient a cording to Fresnel's formula. $\kappa_{A}$ according to my formula, $\kappa_{C}$ according' to Mr. Cunningham's formula, and $\kappa_{Z}$ the value found in Zeeman's experiments. It will be seen that for only one of the four wave-lengths does my formula give a closer approximation to the experimental result thin Mr. Cunningham's.

LXI. The Forms of Planetary Orbnts on the Theory of Relativity. By W. B. Mor'tos, M.A., Queen's Lniversity, Belfast*.

A $\mathrm{N}$ exact integral of the differential equation for a A planetary orbit on Einstein's theory can be expressed, as Prof. Forsyth has pointed out $t$, by use of elliptic functions. The chief interest, of course, attaches to the case actually occurring in astronomy, where the departure from the elliptic orbit of the classical theory is very small. But it is interesting also, if only. from the purely mathematical point of view, to examine the forms which these orbits assume in the most general case. To a certain extent it is easy to foresee how the elliptic form will be further modified as the magnitudes involved are progressively altered. Increasing the strength of the centre will bring about an increase of the apsidal angle. As the velocity in

* Conmunicated by the Author.

† Proc. Roy. Soc. xcrii. p. 145 (1920). 
the orbit is increased we should get something corresponding to the parabolic and hyperbolic paths of the old theory. Of greater interest is the modification which is reached by decreasing the velocity, a direction of change which seems to do less violence to the possibilities of the universe as it is. This is found to lead to orbits of a new type which pasz through the centre and to which, therefore, the name of "captured orbits" may be applied. In the present note the purely mathematical aspect of the matter is alone considered. The co-ordinates $r$ and $\theta$ are taken in their ordinary meanings and curves are drawn to exhibit the relations between them. I leave on one side the question as to the possible bearing of any of the results on observable phenomena in a non-euclidean world where any function of $r$ may be userl instead of $r$.

The first integral of the equation of motion is

$$
\left(\frac{d u}{d \bar{\theta}}\right)^{2}=2 m u^{3}-u^{2}+\frac{2 m}{h^{2}} u+\text { const. }
$$

Here $m$ is the "strength of the centre" or "mass of the sun" in gravitational units and $h$ is twice the area described in unit "proper time" of the planet. It is usual to adopt a system of units in which the constant of gravitation and the velocity of light in space devoid of gravitation are each unity, and the unit of length is the kilometre. The two funclamental unit quantities being regarded as dimensionless constants, the dimensions of mass and leugth become identical and mass also is measured in kilometres. The magnitude of this unit mass is $1 \cdot 35 \times 10^{33} \mathrm{gm}$.

The cubic expression on the right-hand side must be positive in the region of motion and the coefficient of $u^{3}$ is positive. It follows that when all three roots are real the actual values of $u$ must lie between the greatest root and $+\infty$, or else between the middle and smallest roots. If only one root is real, $u$ will lie between this and $+\infty$. Therefore in every case the range of values will be bounded at one end by a root, $i$. e., the motion will include at least one apsidal distance. We shall suppose the particle projected from this apse with varying velocities defined by $h$. Further, if we wish to survey the possible forms of orbits there will be no loss of generality in making the apsidal distance unity. For it is evident from the differential equation that a geometrically similar orbit will be obtained provided $m$ and $h$ are increased in the same ratio as the linear dimensions. Since 
$h_{h}$ is the product of apsidal distance and velocity of projection (measured by unit of proper time), it follows that a path of the same shape is got by projecting with the same velocity at increased distance in presence of a centre of force whos' strength is increased in the same proportion. Accordingly, in what follows $m$ will mean the ratio of the strength of the centre to the initial apsidal distance (both measured, as already explained, in $\mathrm{km}$.), and $h$ will be the ratio of the velocity of projection to the velocity of light.

The first step is to adjust the value of the arbitrary constant, so that $(u-1)$ is a factor of the expression, which then takes the form

$$
2 m(u-1)\left\{u^{2}-\left(\frac{1}{2 m}-1\right) u+\frac{1}{h^{2}}-\left(\frac{1}{2 m}-1\right)\right\},
$$

and the question turns on the nature of the roots of the quadratic factor.

The magnitude of $m$ being fixed, suppose $l$ to increase from zero. There are three critical values of $h_{1-\text { say, }} h_{1}, h_{2}, h_{2}$.

(1) When the roots of the quadratic are equal,

$$
\frac{1}{h_{1}^{2}}=\frac{1}{4}\left(\frac{1}{2 m}+1\right)^{2}-1 \text {. }
$$

(2) When one root is unity,

$$
\frac{1}{h_{2}^{2}}=\frac{1}{m}-3 \text {. }
$$

(3) When one root is zero,

$$
\frac{1}{h_{3}^{2}}=\frac{1}{2 m}-1
$$

The values (1) and (2) coincide when $m=\frac{1}{6},(2)$ and (3) when $m=\frac{1}{4}$, (1) and (3) when $m=\frac{1}{2}$; these are therefore critical values of $m$. The greatest variety in types of orbits is found when $m$ is less than the smallest of these values, so it is convenient to discuss this case first and afterwards to indicate how the results are modified for larger values of $\mathrm{m}$. The politr angle $\theta$ will be measured from the initial apse-line $u=1$. In the integrated equation $u$ is expressed in terms of elliptic functions of the argument $p \theta$, where $p$ is a number for which different expressions are found in the sepurate cases. To illustrate the results obtained I have drawn a series of curves for the case $m=\frac{1}{8}$, for which the critical 

given in the table at the end of the paper. The special values chosen for $\frac{1}{h^{2}}$ are such as to give unbroken values for the modular angle of the elliptic functions in order to facilitate the computations.

\section{Case $m<\frac{1}{6}$.}

(i.) If $h<h_{1}$, then unity is the only real root of the cubic, $u$ runs from 1 to $\infty, r$ from 1 to 0 , and we have a "capture orbit."

Let the result of substituting $u=1$ in the quadratic factor be

$$
\mathrm{H}^{2}=\frac{1}{h^{2}}-\frac{1}{m}+3 ;
$$

the integral is

$$
u=1+\mathrm{H} \cdot \frac{1-\operatorname{cn} p \theta}{1+\operatorname{cn} p \theta}=1+\mathrm{H} \frac{\operatorname{sn}^{2} \frac{1}{2} p \theta \cdot \operatorname{dn}^{2} \frac{1}{2} p \theta}{\mathrm{cn}^{2} \frac{1}{2} p \theta}
$$

wher $p^{2}=2 m \mathrm{H}$ and $k^{2}=\left(\mathrm{H}+\frac{1}{4 m}-\frac{3}{2}\right) / 2 \mathrm{H} . \quad \imath=\infty$ for $\theta=2 \mathrm{~K} / p$, giving the angular range of the motion from the apse to cupture.

As $h$ approaches $h_{1}, \mathrm{H}$ approaches $\left(\frac{1}{4 m}-\frac{3}{2}\right), k$ approaches

Fig. 1.

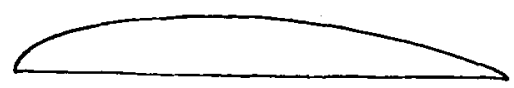

Fig. 2.

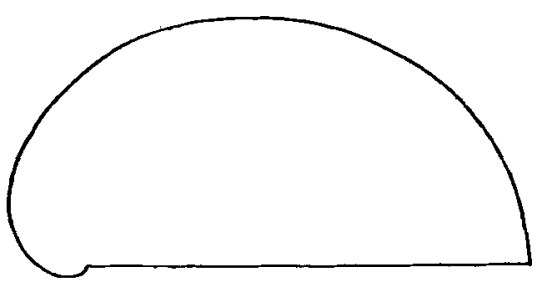

unity, and the particle makes a continually increasing: number of circuits round the centre before being drawn in (figs. 1-4). 
Planetary Orbits on the Theory of Relativity. $\quad 515$

Fig. 3.

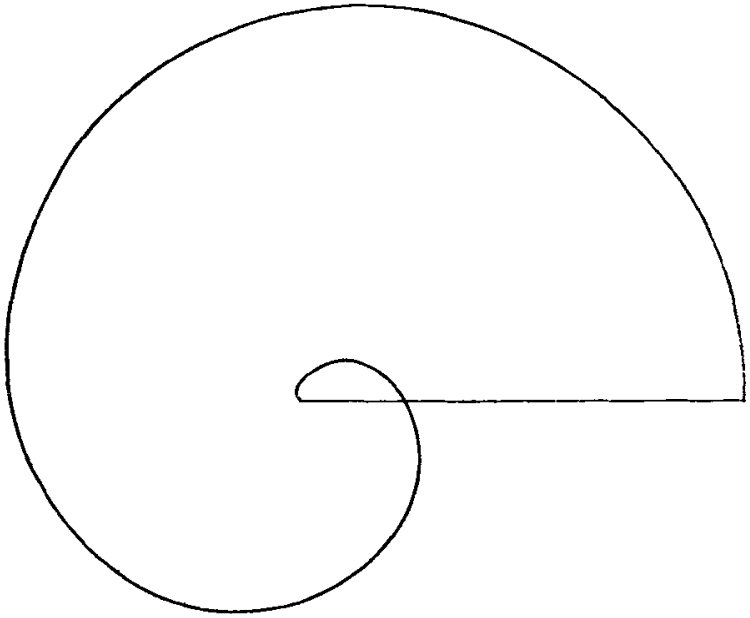

Fig. 4.

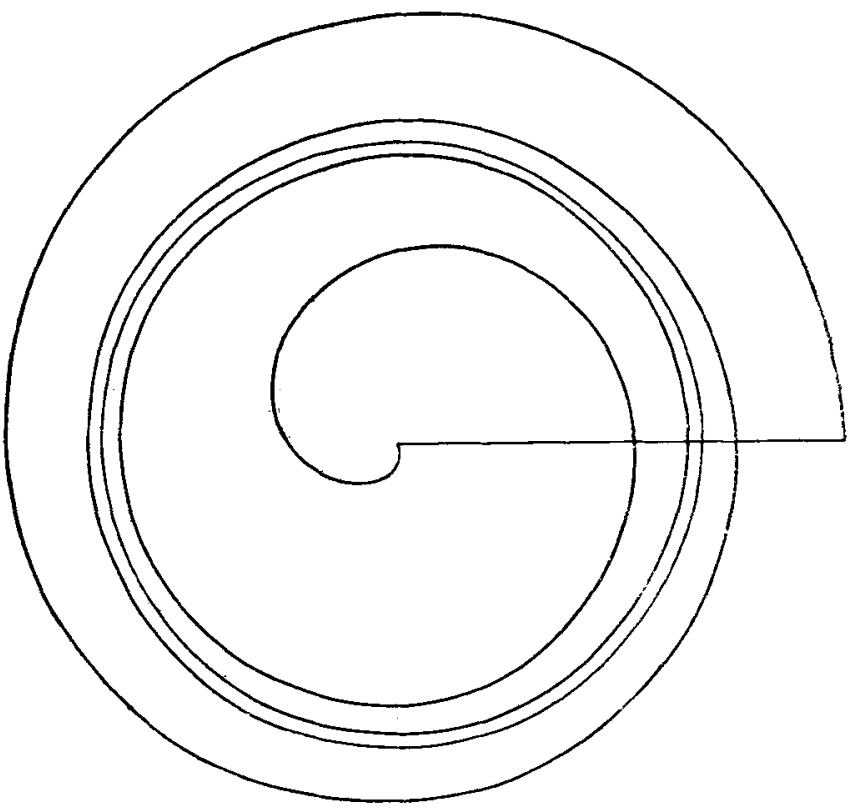

(ii.) When $h=h_{1}$, the elliptic functions become hyperbolic and we have

$$
u=1+\mathrm{H} \tanh ^{2} \frac{1}{2} p \theta \text {. }
$$


As $\theta$ increases $u$ approaches the limiting value

$$
1+\mathrm{H}=\frac{1}{4 m}-\frac{1}{2},
$$

the path continually approximates to a circle of radius $4 \mathrm{~m} /(1-2 \mathrm{~m})$ (fig. 5). This form may be called the "asymptotic circular orbit."

Fig. 5.

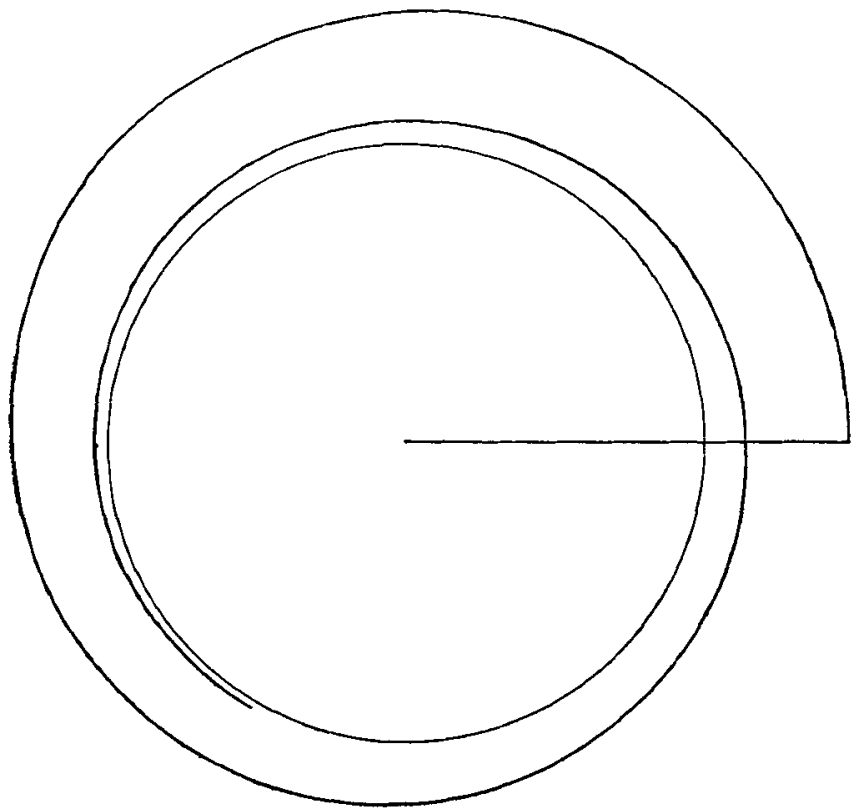

It is not at first sight obvious how this form of orbit can be reached as the limit of class (i.). - On examination it will be seen that, as the number of convolutions increases, the path lingers in the neighbourhood of $u=1+\mathrm{H}$. This is owing to the rapid change in the elliptic function $\mathrm{F}(\phi)$, when $\phi$ and the modular angle are both nearly $90^{\circ}$. As a consequence, a large change in $\theta$ is accompanied by only a small change in $r$ until the case of asymptotic approach to the critical distance is reached.

When $m$ is very small the critical velocity $h_{1}$ may be taken as $4 m$. For the Sun and Earth $m$ is about $10^{-8}$, the Sun's mass being $1.47 \mathrm{~km}$. and the radius of the orbit $1.49 \times 10^{8} \mathrm{~km}$. The critical velocity for capture is $4 \times 10^{-8} \times$ speed of light. $=12$ metres per sec. or about the 2500 th part of the orbital velocity. 
Planetury Orbits on the Theory of Relativity. $\quad 517$ (iii.) $h_{1}<h<h_{2}$.

The quadratic has now two real roots, separated in opposite directions from $\left(\frac{1}{4 m}-\frac{1}{2}\right)$, which is $>1$. Call these $\alpha, \beta$ in descending order, then the arrangement of roots of the cubic is $\alpha, \beta, 1$. The range of values of $u$ is now from 1 to $\beta, i$. e., from aphelion to perihelion. The orbit corresponds to a planetary ellipse with the Einstein advance of the npse. This case may be described as an "elliptic orbit from sphelion" (fig. 6).

Fig. 6.

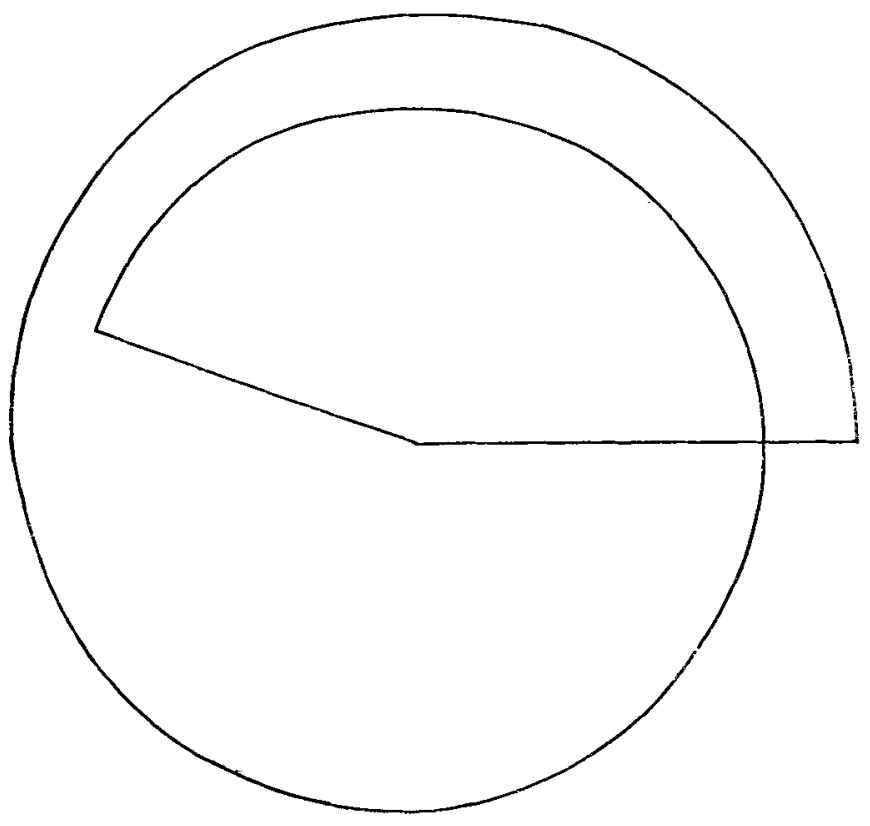

The equition is

$$
u=1+(\beta-1) \cdot \frac{1-\operatorname{cn} p \theta}{1+\operatorname{dn} p \theta}=1+(\beta-1) \sin ^{2} \frac{1}{2}, \theta,
$$

wit ${ }^{1} \quad p^{2}=2 m(\alpha-1), \quad k^{2}=(\beta-1) /(\alpha-1)$.

(In all cases of three real roots the difference of greatest and least roots occurs in $p^{2}$, and the difference of middle and least roots divided by this gives,$i^{2}$ ) The apsidal angle is $2 \mathrm{~K} / p$, decreasing from the intinite value which it has in case (ii.). 
(iv.) When $h=h_{2}$ the $\operatorname{root} \beta=1$, and the orbit is a circle of unit radius (for the sike of continuity in the diagrams this is included as fio. 7). The "velocity in a circle" of unit radius is $m^{\frac{2}{2}} /(1-3 m)^{\frac{1}{2}}$ instead of $m^{\frac{1}{2}}$ as on the ordinary theory.

Fig. 7.

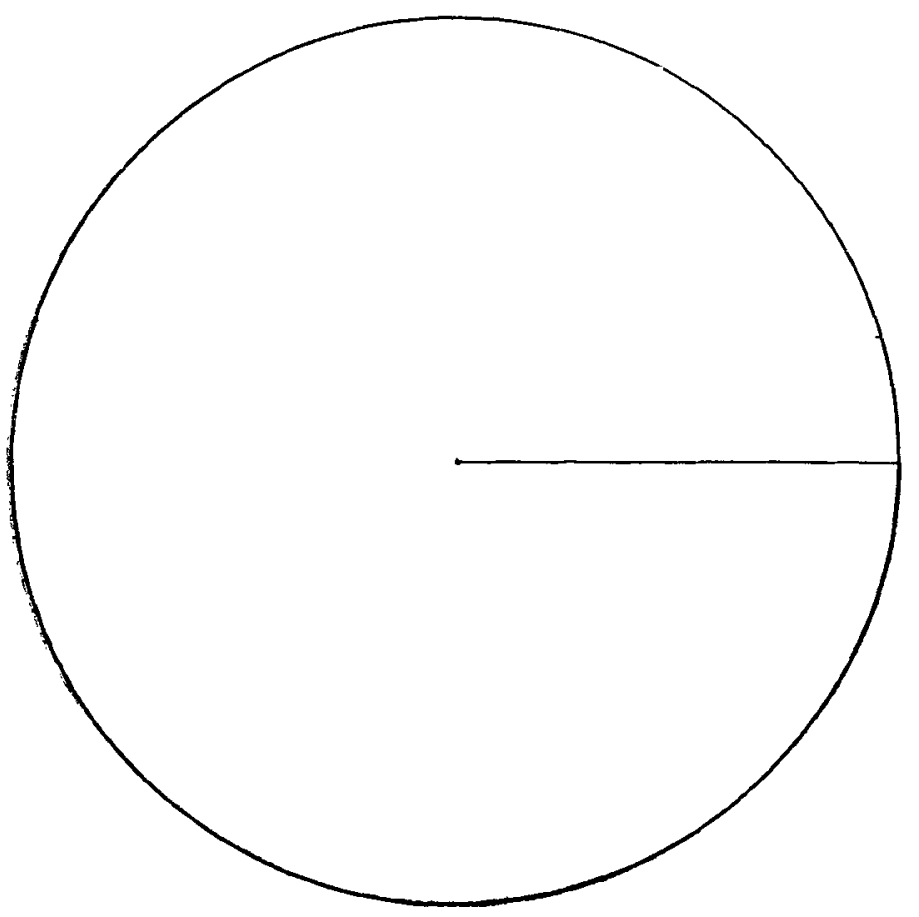

The value of the root $\alpha$ is now $\left(\frac{1}{2 m}-2\right)$. The apsidal angle of the last case approaches $\pi /(1-6 m)^{\frac{1}{2}}$, which replaces $\pi$ as the "apsidal angle of a nearly circular orbit."

(v.) For $h_{2}<h<h_{3}$ the root $\beta$ is less than unity and the arrang $\rightarrow$ ment is $\alpha, 1, \beta ; u$ runs from 1 to $\beta$, and we have an "elliptic orbit from perihelion" (fig. 8). This is the case considered by Prof. Forsyth; his " $\rho$ " is the reciprocal of $p$.

$$
\begin{gathered}
u=\beta+(1-\beta) \cdot \frac{1+\mathrm{cn} p \theta}{1+\operatorname{dn} p \theta}=\beta+(1-\beta) \frac{\mathrm{cn}^{2} \frac{1}{2} p \theta}{\mathrm{dn}^{2} \frac{1}{2} p \theta}, \\
\eta^{2}=2 m(\alpha-\beta), \quad k^{2}=(1-\beta) /(\alpha-\beta) .
\end{gathered}
$$


For purposes of calculation it is more convenient to measure $\theta$ from aphelion and use the second form given under (iii.).

Fig. 8.

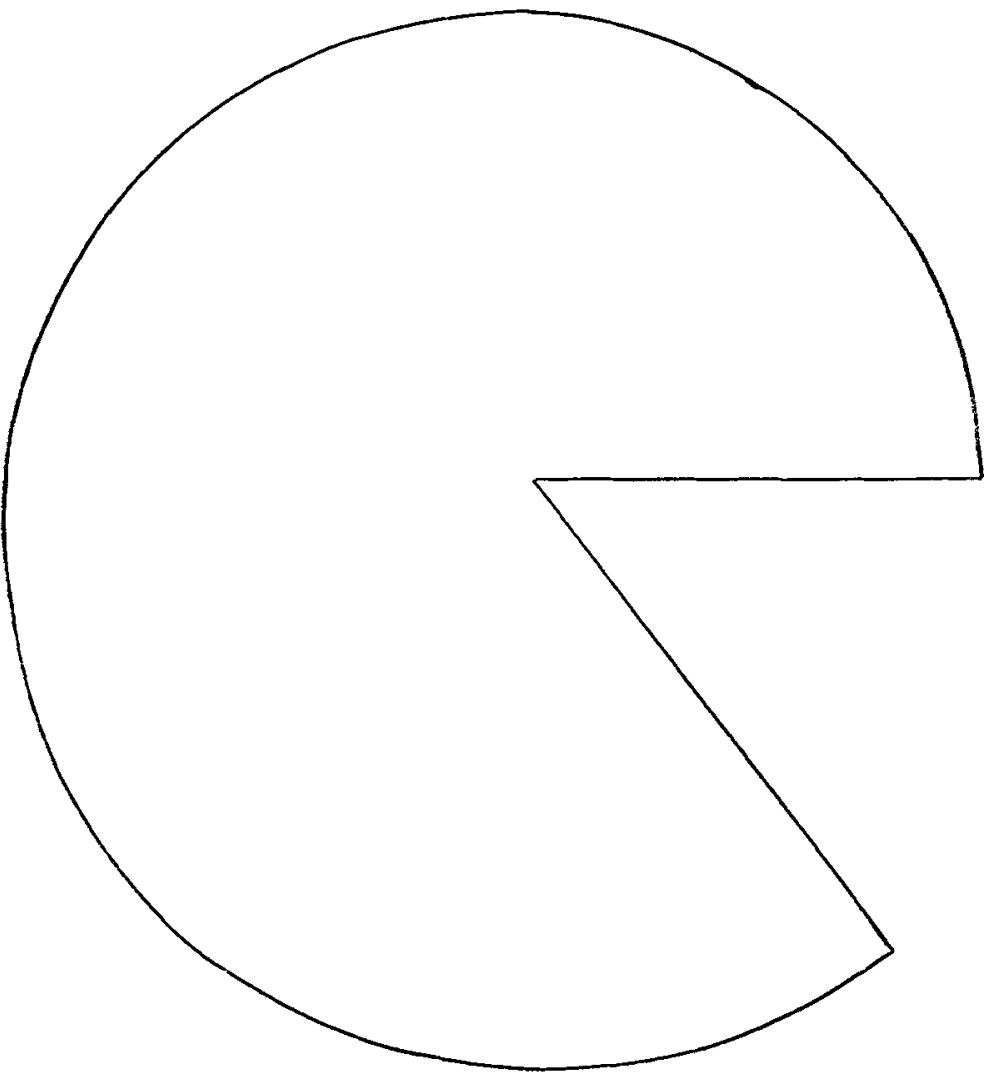

(vi.) $h=h_{3}, \beta=0, \alpha=\frac{1}{2 m}-1$. The aphelion distince becomes infinite and we have the analogue of the ordinary "parabolic orbit." It does not look like a parabola (fig. 9 a), for the two halves of the curve cross each other. The apsidal angle now becomes the angular range of the motion from the apse to infinity. Its value is $2 \mathrm{~K} /(1-2 m)^{\frac{1}{2}}$, with $k^{2}=2 m /(1-2 m)$, and this exceeds $\pi$. The critical velocity for escape to infinity is $(2 m)^{\frac{1}{2}} /(1-2 m)^{\frac{1}{2}}$ instead of $(2 m)^{\frac{1}{2}}$.

(vii.) $h>h_{3}, \beta<0$. The region of motion is now bounded by $u=0$ instead of by $u=\beta$, and the orbits may be classed 
as "hyperbolic." Their equation is that of (v.) with negative $\beta$, but for purposes of calculation it is better to use the form of case (iii.) and write $u=\beta+(1-\beta) \operatorname{sn}^{2} \frac{1}{2} p \theta, \quad p^{2}=2 m(\alpha-\beta), \quad k^{2}=(1-\beta) /(\alpha-\beta)$. $p \theta$ then runs from the value which makes $u=0$ up to $K$, Fig. 9.

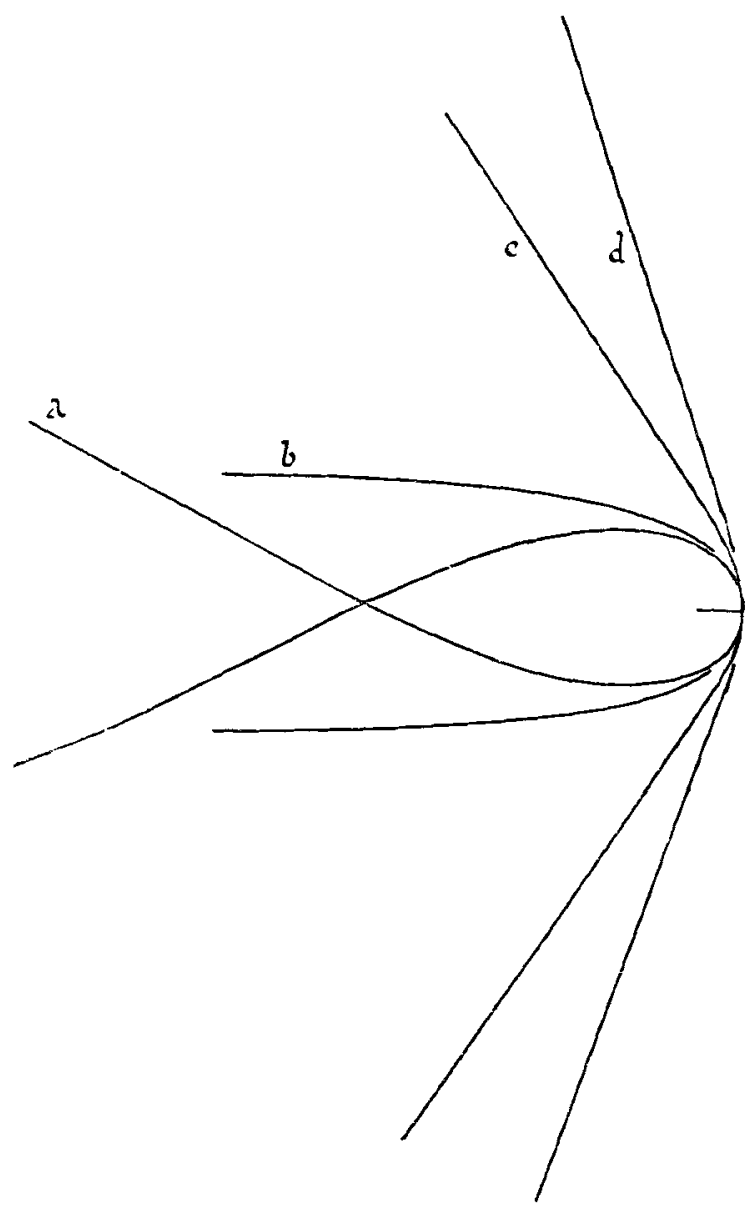

when $u=1$. The angular range from the apse to infinity (or the half-swerve of the orbit increased by $90^{\circ}$ ) is given by

$$
2\left\{\mathrm{~K}-\mathrm{sn}^{-1}(-\beta)^{\frac{1}{2}}(1-\beta)^{-\frac{1}{2}}\right\} / p \text {. }
$$


As the parameter $h$ is increased $\alpha, \beta$ approach limiting values, viz., the roots of the quadratic

$$
u^{2}-\left(\frac{1}{2 m}-1\right) u-\left(\frac{1}{2 m}-1\right)=0,
$$

and so the orbits appronch a definite limiting "hyperbolie" shape. On fig. $9(b),(c),(d)$ are shown three particular cases of orbits in this elass: (b) is the orbit which looks like a parabola, having the angular range between apse and infinity $=\pi$, (c) is that for which $h=1$, and $(d)$ is the limiting form for $l=\infty$, all drawn for $m=\frac{1}{8}$. The scale of fig. 9 is 1 io that of the others.

\section{Modifications for larger values of $m$.}

As $m$ approaclies $\frac{1}{6}$ the radius of the asymptotic circle becomes unity and the orbit becomes identical with the ordinary circular orbit ; the "elliptic orbits from aphelion" are squeezed out. The later forms follow the same sequence as betore.

When $\frac{1}{6}<m<\frac{1}{4}$ for $h_{l}=h_{1}$ the quadratic has a double root $\left(\begin{array}{c}1 \\ 4 m\end{array}-\frac{1}{2}\right)$, which is now less than unity. Therefore, $u$ runs from 1 to $\infty$ in stages (ii.) and (iii.) as well as in stage (i.); the captured orbits extend over the first three classes. The positive quantity $H$ now approaches $\left(\frac{3}{2}-\frac{1}{4 m}\right)$ and $k^{2}$ approaches zero, so the elliptic functions become circular insteal of hyperbolic. The equation for (ii.) is

$$
u=1+H \tan ^{2} \frac{1}{2} p \theta
$$

so the centre is reached at $\theta=\pi / p$, where $p^{2}=2 m \mathrm{H}$.

In case (iii.) the arrangement of the roots of the cubic is $1, \alpha, \beta$. The integral is

$u=\alpha+(1-\alpha) / \mathrm{cn}^{2} \frac{1}{2} p \theta, \quad l^{2}=(\alpha-\beta) /(1-\beta), \quad p^{2}=2 m(1-\beta)$.

Thus $u=\infty$ for $\theta=2 \mathrm{~K} / p$. When $\alpha=1$ the circular orbit is reached and the succeeding forms are as before.

For $\frac{1}{4}<m<\frac{1}{2}$ there is a further extension of the range of captured orbits. The critical values $l_{2}$ and $h_{3}$ now change places, $h_{2}>l_{3}$. All paths get to the centre until $h_{l}=h_{12}$, the orbit is then circular and succeding paths are hy perbolic.

For $m>\frac{1}{2}$ the roots of the quadratic are always imaginary, so that all orbits are captured.

These results may be summarised in tabular form, the Plil. Mag. S. 6. Vol. 42. No. 250. Oct. 1921. $2 \mathrm{~N}$ 
522 Forms of Planetary Orlits on Theory of Relativity.

character of the orbit being set against the corresponding value or interval of the parameter $h:-$

$$
\begin{aligned}
& \begin{array}{l|l}
\text { h. } & m<\frac{1}{6} . \\
\end{array} \\
& \text { Captured. } \\
& h_{1} \text { Asymptotic circular. } \\
& \text { Elliptic from aphelion. } \\
& \text { h. Circular. } \\
& \text { Elliptic from perihelion. } \\
& \text { 7. Parabolic. } \\
& \text { Hyperbolic. } \\
& \infty \\
& 0 \quad \underline{\frac{1}{4}<m<\frac{1}{2}} . \\
& \left.\begin{array}{l}
h_{1} \\
h_{3}
\end{array}\right\} \text { Captured. } \\
& h_{2}^{3} \text { Circular. } \\
& \text { Hyperbolic. } \\
& m>\frac{1}{2}
\end{aligned}
$$

\begin{tabular}{|c|c|c|c|c|c|}
\hline Fig. & $1 / h^{2}$. & $h$. & Ratio. & $\begin{array}{c}\text { Angle } \\
\text { (degrees). }\end{array}$ & Orbit. \\
\hline 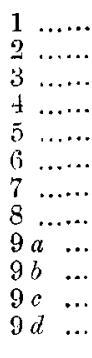 & $\begin{array}{l}210 \cdot 3 \\
13 \cdot 29 \\
6 \\
5 \cdot 2503 \\
51 \\
5 \frac{2}{6} \\
5 \\
41 \frac{1}{16} \\
3 \\
2 \cdot 64 \\
1 \\
0\end{array}$ & $\begin{array}{l}\cdot 069 \\
.271 \\
.408 \\
.43649 \\
.43644 \\
.438 \\
\cdot \pm 47 \\
.462 \\
.577 \\
.616 \\
1 \\
\infty\end{array}$ & $\begin{array}{l}0 \\
0 \\
0 \\
0 \\
\frac{2}{3} \\
\frac{3}{4} \\
1 \\
\frac{4}{3} \\
\infty \\
\infty \\
\infty \\
\infty\end{array}$ & $\begin{array}{c}113 \cdot 1 \\
261 \cdot 4 \\
360+134 \cdot 2 \\
4 \times 360+321 \\
\infty \\
360+160 \cdot 4 \\
360 \\
307 \cdot 5 \\
229 \cdot 4 \\
180 \\
1245 \\
109 \cdot 2\end{array}$ & $\begin{array}{l}\text { Captured. } \\
\text { Asymptotic circular. } \\
\text { Elliptic from aphelion. } \\
\text { Circular. } \\
\text { Elliptic from peribelion. } \\
\text { Parabolic. } \\
\text { Hyperbolic. }\end{array}$ \\
\hline
\end{tabular}

Elliptic from perihelion.

It appears that the circular form may be called stable only for $m<\frac{1}{9}$. For $\frac{1}{8}<m<\frac{1}{6}$ it is stable only on the side of increased velocity, and beyond these limits it is unstable on both sides.

\section{Description of Diagrams.}

The following table gives details about the curves, plotted for $m=\frac{1}{3}$. The first and second columns gire $1 / h^{2}$ and $h$. The third, headed "ratio," gives the ratio of the other extreme distance to the initial distance, which has been taken as the unit in the above work. The "angle" column gives the angular range from the initial line to capture, to the other apse, or to infinity, as the case may be :- 